\title{
Radiative improvement of the lattice NRQCD action using the background field method and application to the hyperfine splitting of quarkonium states
}

\author{
T. C. Hammant, ${ }^{1}$ A. G. Hart, ${ }^{2,3}$ G. M. von Hippel, ${ }^{4}$ R. R. Horgan, ${ }^{1}$ and C. J. Monahan ${ }^{1}$ \\ ${ }^{1}$ Department of Applied Mathematics and Theoretical Physics, University of Cambridge, \\ Centre for Mathematical Sciences, Cambridge CB3 OWA, United Kingdom \\ ${ }^{2}$ Cray Exascale Research Initiative Europe, JCMB, King's Buildings, Edinburgh EH9 3JZ, United Kingdom \\ ${ }^{3}$ SUPA, School of Physics and Astronomy, University of Edinburgh, King's Buildings, Edinburgh EH9 3JZ, United Kingdom \\ ${ }^{4}$ Institut für Kernphysik, University of Mainz, Becher-Weg 45, 55099 Mainz, Germany
}

\begin{abstract}
We present the first application of the background field method to Non-Relativistic QCD (NRQCD) on the lattice in order to determine the one-loop radiative corrections to the coefficients of the NRQCD action in a manifestly gauge-covariant manner. The coefficient of the $\boldsymbol{\sigma} \cdot \boldsymbol{B}$ term in the NRQCD action is computed at the one-loop level; the resulting shift of the hyperfine splitting of bottomonium is found to bring the lattice predictions in line with experiment.
\end{abstract}

PACS numbers: $12.38 . \mathrm{Bx}, 12.38 . \mathrm{Gc}$

Non-Relativistic QCD (NRQCD) [1] is an effective field theory that has been applied with considerable success to the description of hadrons containing heavy quarks [2]. However, the currently used NRQCD actions do not include radiative improvement (with the exception of tadpole improvement), and this will affect the precision with which crucial quantities such as the hyperfine splitting between the $\Upsilon$ and the $\eta_{\mathrm{b}}$ can be determined [3]. In contrast to this, Non-Relativistic QED (NRQED) has been successfully improved and applied to obtain highly precise theoretical predictions for the fine structure of muonium [4, 5]. It is clearly highly desirable to improve NRQCD in a similar manner. This is rendered complicated by the nature of the non-abelian gauge interactions in QCD and NRQCD, which requires NRQCD to be implemented on a lattice and hence makes it necessary to retain the full $1 /(m a)^{n}$ dependences, whereas in continuum NRQED $(\Lambda / m)^{n}$ terms can be omitted in a consistent manner. Moreover, IR divergences play a non-trivial rôle in QCD and NRQCD.

In this letter, we present the first calculation of radiative corrections to coefficients in the lattice NRQCD action using the background field (BF) method. We compute the one-loop effective action in lattice NRQCD and match it term-by-term to the non-relativistic reduction of the oneloop effective action for continuum QCD. In particular, we determine the one-loop corrections to the coefficient of the chromomagnetic $\boldsymbol{\sigma} \cdot \boldsymbol{B}$ term and the four-fermion spin-spin interaction; these corrections are important for the accurate calculation of the hyperfine structure of heavy quark states using NRQCD.

\section{THE BACKGROUND FIELD METHOD FOR LATTICE NRQCD}

The BF method [6-9] is a well-established tool to compute the effective action in quantum field theory. The auxiliary gauge invariance of BFG amplitudes implies that the effective action contains only gauge-covariant operators which leads to a set of Ward Identities in QCD that reduce the amount of calculation necessary to renormalize the theory. This property is important for operators of dimension $D>4$ where the loss of gauge-covariance would lead to a proliferation of additional operators and is vital to the radiative improvement of NRQCD which is a non-relativistic expansion on operators of increasing dimension; only BFG will guarantee the gauge covariance of the improved effective action. Whilst the presence of gauge non-covariant finite terms with $D>4$ in the effective action is not per se incorrect, they obscure the underlying gauge symmetry and greatly complicate the theory and simulation. An attempt to match without using BFG would lead to the appearance of ultraviolet logarithms, which would have to be cancelled by the contributions from additional non-gauge-covariant operators. Although BFG does not guarantee that the coefficients in the effective action are independent of the gauge parameter [10], in our case we match between theories using on-shell quantities and we explicitly find that the coefficients are independent of the gauge parameter in both QCD and NRQCD. Moreover, the QED-like Ward identities in BFG imply that the one-particle irreducible (1PI) vertex functions are finite, and that the coupling $g$ is renormalized only by the contribution from the gluonic self-energy, whereas the $\mathrm{BF}$ is not renormalized. This is true both in QCD and NRQCD, which allows us to match the theories by equating two finite quantities. As a consequence of this crucial property of BFG, we are free to use different regulators in QCD and NRQCD. In particular, we can calculate the QCD vertex analytically in the continuum using dimensional regularization, or on a fine lattice and taking the continuum limit. The latter is particularly convenient for checking the gauge-parameter independence of the result, since the analytical calculation becomes rather involved for general values of the gauge parameter.

In the following we denote the perturbative expansion for a generic parameter $w$ as $w=\sum_{n=0} w^{(n)} \alpha^{n}$. 

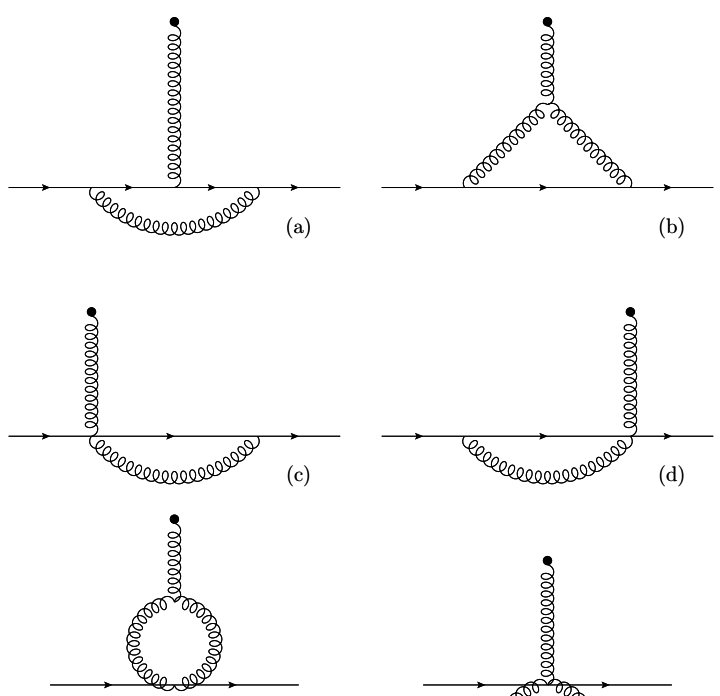

(e)

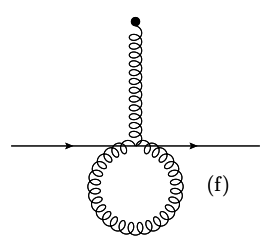

FIG. 1. Feynman diagrams to be computed in both QCD and NRQCD for matching the $\boldsymbol{\sigma} \cdot \boldsymbol{B}$ term in the NRQCD action.

\section{MATCHING THE $\sigma \cdot B$ TERM}

The effective action for continuum QCD contains the following terms involving the fermion fields:

$$
\Gamma[\Psi, \bar{\Psi}, A]=Z_{2}^{-1} \bar{\Psi} \not D \Psi+\delta Z_{\sigma} \bar{\Psi} \frac{\sigma^{\mu \nu} F_{\mu \nu}}{2 m} \Psi+\ldots
$$

which after renormalization of the first term gives

$$
\Gamma\left[\Psi_{R}, \bar{\Psi}_{R}, A\right]=\bar{\Psi}_{R} \not D \Psi_{R}+b_{\sigma} \bar{\Psi}_{R} \frac{\sigma^{\mu \nu} F_{\mu \nu}}{2 m_{R}} \Psi_{R}+\ldots
$$

with

$$
b_{\sigma}=\delta Z_{\sigma} Z_{2} Z_{m}=\sum_{n=1} b_{\sigma}^{(n)} \alpha^{n}
$$

where the leading correction is of order $O\left(\alpha_{s}\right)$ and comes from $\delta Z_{\sigma}$ alone. After performing the non-relativistic reduction by a Foldy-Wouthuysen-Tani (FWT) transformation, we find that the term relevant for the determination of the chromomagnetic moment of the quark is

$$
\left(1+b_{\sigma}\right) \psi_{R}^{\dagger} \frac{\boldsymbol{\sigma} \cdot \boldsymbol{B}}{2 m_{R}} \psi_{R}
$$

A straightforward analytical calculation of the Feynman diagrams shown in figure 1 (a)-(b) gives

$$
b_{\sigma}=\left(\frac{3}{2 \pi} \log \frac{\mu}{m}+\frac{13}{6 \pi}\right) \alpha
$$

at the one-loop level, where $\mu$ is the infrared cutoff.

The effective action for NRQCD contains the spindependent term

$$
\Gamma_{\sigma}\left[\psi, \psi^{\dagger}, A\right]=c_{4} Z_{\sigma}^{\mathrm{NR}} \psi^{\dagger} \frac{\boldsymbol{\sigma} \cdot \boldsymbol{B}}{2 M} \psi
$$

which after renormalization becomes

$$
\Gamma_{\sigma}\left[\psi_{R}, \psi_{R}^{\dagger}, A\right]=c_{4} Z_{\sigma}^{\mathrm{NR}} Z_{2}^{\mathrm{NR}} Z_{m}^{\mathrm{NR}} \psi_{R}^{\dagger} \frac{\boldsymbol{\sigma} \cdot \boldsymbol{B}}{2 M_{R}} \psi_{R} .
$$

We require that the anomalous chromomagnetic moment in QCD and NRQCD be equal and find the matching condition

$$
c_{4} Z_{\sigma}^{\mathrm{NR}} Z_{2}^{\mathrm{NR}} Z_{m}^{\mathrm{NR}}=1+b_{\sigma}
$$

and at tree level and one-loop order we find

$$
\begin{aligned}
& c_{4}^{(0)}=1, \\
& c_{4}^{(1)}=b_{\sigma}^{(1)}-\delta Z_{\sigma}^{\mathrm{NR},(1)}-\delta Z_{2}^{\mathrm{NR},(1)}-\delta Z_{m}^{\mathrm{NR},(1)} .
\end{aligned}
$$

The NRQCD contribution to $c_{4}^{(1)}$ contains a logarithmic IR divergence $\frac{3 \alpha}{2 \pi} \log (\mu a)$, which combines with the IR logarithm from the QCD result above to yield an overall logarithmic contribution $-\frac{3 \alpha}{2 \pi} \log (M a)$.

Besides the ordinary diagrammatic contributions calculated below, we also need to take into account the contributions from the mean-field improvement $U \mapsto U / u_{0}$, which affect $\delta Z_{\sigma}^{\mathrm{NR},(1)}$ and $\delta Z_{m}^{\mathrm{NR},(1)}$. Perturbatively, $u_{0}=$ $1-\alpha_{s} u_{0}^{(2)}$, and the contributions from inserting this expansion into the NRQCD action can be worked out algebraically. The final result for the one-loop correction to $c_{4}$ is then

$$
\begin{aligned}
c_{4}^{(1)} & =\frac{13}{6 \pi}-\delta \tilde{Z}_{\sigma}^{\mathrm{NR},(1)}-\delta \tilde{Z}_{2}^{\mathrm{NR},(1)}-\delta \tilde{Z}_{m}^{\mathrm{NR},(1)} \\
& -\delta Z_{m}^{\mathrm{tad},(1)}-\delta Z_{\sigma}^{\mathrm{tad},(1)}-\frac{3}{2 \pi} \log M a
\end{aligned}
$$

where $\delta \tilde{Z}_{X}$ denotes a finite diagrammatic contribution. We expect the coefficient $c_{4}$ to be gauge-parameter independent for on-shell quarks, since it is directly related to the hyperfine splitting, which is a physical quantity.

\section{THE FOUR-FERMION SPIN-SPIN INTERACTION}

In NRQCD the hyperfine splitting in the $b \bar{b}$ system also receives a contribution from the spin-dependent fourfermion operators generated by $Q \bar{Q} \rightarrow Q \bar{Q}$ scattering in the colour singlet channel. It is conventional to write these contributions using a Fierz transformation [1, 11]

$S_{4 f}=d_{1} \frac{\alpha^{2}}{M^{2}}\left(\psi^{\dagger} \chi^{*}\right)\left(\chi^{T} \psi\right)+d_{2} \frac{\alpha^{2}}{M^{2}}\left(\psi^{\dagger} \boldsymbol{\sigma} \chi^{*}\right) \cdot\left(\chi^{T} \boldsymbol{\sigma} \psi\right)$,

where $\psi$ and $\chi$ are the quark and anti-quark fields, respectively, treated as different particle species with corresponding representations of their spin and colour algebras. The spin-independent contributions to $d_{1}$ and $d_{2}$ from $Q \bar{Q}$ scattering are not included as they do not influence the hyperfine structure. In QCD the two continuum diagrams are shown in figures 2(a) and 2(b), and in NRQCD all diagrams 
in figure 2 need to be calculated. The one-loop contributions to the renormalization constants for the operators in eqn. (11) take the form, respectively,

$$
\begin{aligned}
& Z_{f 1}=\alpha^{2}\left(A_{f 1}-\log \frac{\mu}{m}-\frac{16 \pi}{27} \frac{m}{\mu}\right), \\
& Z_{f 2}=-\frac{1}{3} Z_{f 1} .
\end{aligned}
$$

The last term in both expressions is the Coulomb singularity arising from the Coulomb gluon exchange in figure 2(a). For QCD these expressions were verified numerically and for both QCD and NRQCD were shown to be gaugeparameter independent; there are two independent colour trace combinations, each of which is separately gauge independent. In the numerical calculations we used IR subtraction functions to analytically remove both IR and Coulomb divergences; this greatly improved convergence. For QCD we find

$$
A_{f 1}^{R}=\frac{8}{9} .
$$

The matching parameters for the term in the NRQCD action, including the two-gluon annihilation contribution to $d_{1}$ [11], are then

$$
\begin{aligned}
& d_{1}=-3 d_{2}-\frac{2}{9}(2-2 \log 2) \\
& d_{2}=-\frac{8}{27}+\frac{1}{3} A_{f 1}^{N R}-\frac{1}{3} \log M a .
\end{aligned}
$$

\section{IMPLEMENTATION AND RESULTS}

To perform the calculation in NRQCD, we employ the HIPPY and HPSRC packages for automated lattice perturbation theory [12, 13], which we extended to deal with the modifications of the usual Feynman rules engendered by the use of BFG [14, 15]. Specifically, in the expansion of a gauge link the background fields $B_{\mu}$ must always appear to the right of the quantum fields $A_{\mu}$ and so not all orderings of background and quantum fields can arise. Additional contributions to all purely gluonic vertices including exactly two quantum gluons arise from the gauge-fixing term and additional ghost field vertices are generated which have been included but are not needed for the present calculation. For further implementation details the reader is referred to [16].

For the $\boldsymbol{\sigma} \cdot \boldsymbol{B}$ operator matching we compute the diagrams in figures 1 (a)-(f) and for the four-fermion operator matching we compute the diagrams in figure 2 We use the HPSRC library, which includes a parallel implementation of VEGAS [17], as well as routines for automatic differentiation of Feynman diagrams [18]. We carried out a number of checks of the calculation. Firstly, we replicate the known IR logs correctly. We find that the coefficients of these logs are gauge-parameter independent and, since this is not true of the contributions from individual diagrams, it provides

\begin{tabular}{|c|ccc|}
\hline$M a$ & 1.95 & 2.8 & 4.0 \\
\hline \hline$\delta \tilde{Z}_{\sigma}+\delta \tilde{Z}_{2}$ & $-5.164(7)$ & $-4.913(6)$ & $-4.739(6)$ \\
$\delta \tilde{Z}_{m}$ & $1.512(1)$ & $1.022(3)$ & $0.723(2)$ \\
$\delta Z_{\sigma}^{\text {tad }}$ & 4.387 & 4.077 & 3.841 \\
$\delta Z_{m}^{\text {tad }}$ & -1.092 & -0.787 & -0.641 \\
$c_{4}^{(1)}$ & $0.728(7)$ & $0.799(7)$ & $0.842(6)$ \\
$d_{1}$ & $0.638(7)$ & $-0.109(14)$ & $-1.138(25)$ \\
$d_{2}$ & $-0.258(2)$ & $-0.009(5)$ & $0.334(8)$ \\
\hline
\end{tabular}

TABLE I. Renormalization parameters of the $\boldsymbol{\sigma} \cdot \boldsymbol{B}$ and the fourfermion terms defined, respectively, in eqns. (11) and (14).

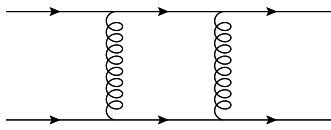

(a)

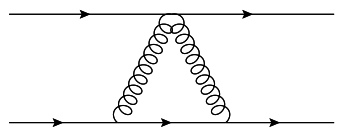

(c)

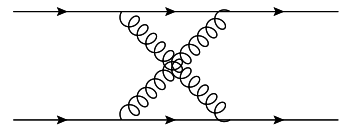

(b)

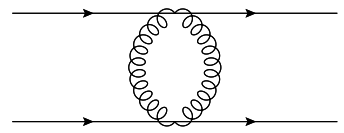

(d)
FIG. 2. Feynman diagrams to be computed in both QCD and NRQCD for matching the four-fermion terms in the NRQCD action. There are two diagrams with the topology of (c).

a strong check. Second, we check that the non-logarithmic part of the result is similarly gauge-parameter independent where the individual contributions are not. For matching the four-fermion terms it is vital to employ IR subtraction functions to remove logarithmic and Coulomb IR singularities.

For NRQCD, we used the action from [2] with stability parameter $n=2$, and we used the Symanzik improved gluon action [19], which were also used by the MILC collaboration [20] whose configurations were used in [2]. We find

$$
\delta Z_{m}^{\mathrm{tad},(1)}=-\left(\frac{2}{3}+\frac{3}{(M a)^{2}}\right) \alpha_{s} u_{0}^{(2)}
$$

The tadpole contribution to $\delta Z_{\sigma}^{\mathrm{NR},(1)}$ comes from the meanfield improvement of the improved field-strength tensor and from the cross-multiplication of the tree-level $\boldsymbol{\sigma} \cdot \boldsymbol{B}$ term with the tadpole corrections terms in $H_{0}$ [2]. The overall result is

$$
\delta Z_{\sigma}^{\mathrm{tad},(1)}=\left(\frac{13}{3}+\frac{13}{4 M a}-\frac{3}{8 n(M a)^{2}}-\frac{3}{4(M a)^{3}}\right) \cdot u_{0}^{(2)} .
$$

We chose the Landau mean link to be $u_{0}^{(2)}=0.750$ [21]. Our results are shown in table I] Whilst there is no substitute for including these radiative corrections in a simulation, we note that both operators give a contribution to the hyperfine splitting that is dominated by a contact term. The leading contribution from the $g \boldsymbol{\sigma} \cdot \boldsymbol{B}$ term, already included in the simulation, is $O\left(\alpha_{s}\right)$ and so the radiative 


\begin{tabular}{|cc|cc|c|c|}
\hline & & \multicolumn{2}{|c|}{ Correction \% } & hfs $(\mathrm{MeV})$ & hfs $(\mathrm{MeV})$ \\
$M a$ & $\alpha_{V}\left(q^{*}\right)$ & 4-fermion & $\sigma \cdot B$ & ref. [2] & corrected \\
\hline \hline 1.95 & 0.216 & $-10.3(1)$ & $+31.4(3)$ & $56(2)$ & $68(3)(5)(6)$ \\
2.8 & 0.249 & $+1.3(2)$ & $+39.8(3)$ & $50(2)$ & $71(3)(6)(5)$ \\
4.0 & 0.293 & $+23.2(4)$ & $+49.3(3)$ & $41(2)$ & $71(3)(7)(4)$ \\
\hline
\end{tabular}

TABLE II. Corrections to the bottomonium hyperfine splitting results of [2] arising from the radiative improvement of the action. In the last column the errors are statistical, $O\left(\alpha^{2}\right)$, and relativistic corrections.

correction to $c_{4}$ and the leading contribution from the fourfermion terms in eqn. (11) both contribute at $O\left(\alpha^{2}\right)$ giving an $O(\alpha)$ correction to the measured tree-level contribution. A reasonable estimate for the multiplicative correction to the tree-level prediction for the hyperfine splitting is then

$$
1+\alpha_{V}\left(q^{*}\right)\left(2 c_{4}^{(1)}-\frac{27}{16 \pi}\left(d_{1}-d_{2}\right)\right)
$$

where we chose $q^{*}=\pi / a$. Applying our results to the hyperfine splitting of bottomonium, we find the corrections given in table $\Pi$ ] for the data points of [2]. On all lattices the correction is positive and the remaining $O\left(a^{2}\right)$ error in the NRQCD predictions of [2] is reduced to be within errors.

\section{CONCLUSION}

In this letter, we have applied the BF method to lattice NRQCD for the first time and have computed the one-loop radiative correction to the coefficient, $c_{4}$, of the $\boldsymbol{\sigma} \cdot \boldsymbol{B}$ operator and the one-loop radiative contribution to the coefficients, $d_{1}$ and $d_{2}$ of the four-fermion contact operators that affect the hyperfine structure of heavy quark mesons. The gauge independence of our calculation was explicitly checked by carrying out both relativistic and nonrelativistic calculations in the lattice theory. This is possible because in BFG all calculations are UV finite. Our results are summarized in table $\mathrm{I}$ and in eqns. (11) and (14). In particular, in eqn. (11) there is a negative correction to $c_{4}$ due the IR divergences. However, it turns out that the constant terms more than cancel this effect and the correction to $c_{4}$ is positive. Whilst there is no substitute for including these corrections in a simulation, we have given an estimate for the correction to the $\Upsilon-\eta_{b}$ hyperfine splitting measured by Gray et al. [2] in table III. The result is to reduce the lattice spacing dependence to within errors and to give an estimate for this hyperfine splitting of $68(3)(5)(6) \mathrm{MeV}$ to be compared with the experimental value of $69.3(2.8) \mathrm{MeV}$ [22]. The errors shown are statistical, $O\left(\alpha^{2}\right)$, and due to relativistic corrections, respectively. The elimination of $O\left(\alpha a^{2}\right)$ errors and the agreement with experiment gives us confidence that the calculations are robust.
The determination of the one-loop radiative corrections to the coefficients of the $\boldsymbol{p}^{4}$, Darwin and spin-orbit terms and other four-fermion contact terms, as well as more details of the calculations will be presented in a longer paper in the near future.

We thank Alan Gray, Andrew Lee, Christine Davies and Matthew Wingate for useful discussions. We thank the DEISA Consortium, co-funded through the EU FP6 project RI-031513 and the FP7 project RI-222919, for support within the DEISA Extreme Computing Initiative. This work was supported by STFC under grants ST/G000581/1 and ST/H008861/1. The calculations for this work were, in part, performed on the University of Cambridge HPCs as a component of the DiRAC facility jointly funded by STFC and the Large Facilities Capital Fund of BIS. The University of Edinburgh is supported in part by the Scottish Universities Physics Alliance (SUPA). AH was supported in part by the Royal Society (UK).

[1] G. P. Lepage et al., Phys.Rev. D46, 4052 (1992), [heplat/9205007].

[2] A. Gray et al., Phys.Rev. D72, 094507 (2005), [heplat/0507013].

[3] S. Meinel, Phys.Rev. D82, 114502 (2010), [1007.3966].

[4] T. Kinoshita and M. Nio, Phys.Rev. D53, 4909 (1996), [hep$\mathrm{ph} / 9512327]$.

[5] M. Nio and T. Kinoshita, Phys.Rev. D55, 7267 (1997), [hep$\mathrm{ph} / 9702218]$.

[6] L. Abbott, M. Grisaru and R. Schaefer, Nucl.Phys. B229, 372 (1983).

[7] B. S. DeWitt, Phys.Rev. 162, 1195 (1967).

[8] B. S. DeWitt, Phys.Rev. 162, 1239 (1967).

[9] H. Kluberg-Stern and J. Zuber, Phys.Rev. D12, 482 (1975).

[10] A. Rebhan, Nucl.Phys. B288, 832 (1987).

[11] P. Labelle, S. M. Zebarjad and C. P. Burgess, Phys. Rev. D56, 267 (1997), [hep-ph/9611313].

[12] A. Hart, G. M. von Hippel, R. R. Horgan and L. C. Storoni, J.Comput.Phys. 209, 340 (2005), [hep-lat/0411026].

[13] A. Hart, G. M. von Hippel, R. R. Horgan and E. H. Müller, Comput.Phys.Commun. 180, 2698 (2009), [0904.0375].

[14] M. Lüscher and P. Weisz, Nucl.Phys. B452, 213 (1995), [hep-lat/9504006].

[15] M. Lüscher and P. Weisz, Nucl.Phys. B452, 234 (1995), [hep-lat/9505011].

[16] T. C. Hammant et al., PoS LATTICE2010, 043 (2010), [1011.2696].

[17] G. Lepage, J.Comput.Phys. 27, 192 (1978).

[18] G. M. von Hippel, Comput.Phys.Commun. 181, 705 (2010), [0910.5111].

[19] M. Lüscher and P. Weisz, Nucl.Phys. B266, 309 (1986).

[20] C. Bernard et al., Phys.Rev. D64, 054506 (2001), [heplat/0104002].

[21] M. A. Nobes et al., hep-lat/0110051.

[22] K. Nakamura et al., J. Phys. G 37, 075021 (2010). 